\title{
High-energy electron relaxation and full-band electron dynamics in aluminium nitride
}

\author{
C. Bulutay ${ }^{\mathrm{a}}$, B.K. Ridley ${ }^{\mathrm{b}, *}$, N.A. Zakhleniuk ${ }^{\mathrm{c}}$ \\ a Department of Physics, Bilkent University, 06533 Bilkent, Ankara, Turkey \\ ${ }^{\mathrm{b}}$ Department of Electronic Systems Engineering, University of Essex, Wivenhoe Park, Colchester C04 3SQ, UK \\ ${ }^{\mathrm{c}}$ Marconi Optical Components, Caswell, Towcester, Northants, NN12 8EQ, UK
}

\begin{abstract}
Material properties of AlN, particularly its wide band gap around $6 \mathrm{eV}$, warrant its operation in the high-field transport regimes reaching $\mathrm{MV} / \mathrm{cm}$ fields. In this theoretical work, we examine the full-band scattering of conduction band electrons in AlN due to polar optical phonon (POP) emission, which is the main scattering channel at high fields. First, we obtain the band structure for the wurtzite phase of AIN using the empirical pseudopotential method. Scattering rates along the full length of several high-symmetry directions are computed efficiently through the Lehmann-Taut Brillouin zone integration technique. In order to shed light on the behaviour of the velocity-field characteristics at extremely high electric fields, in the order of a few $\mathrm{MV} / \mathrm{cm}$, we resort to an Esaki-Tsu estimation. Comparison of these results for AlN is made with our similar work on GaN. With typically more than $50 \%$ higher POP scattering rate compared to GaN, AlN has poorer high-field prospects. Availability of these data for AlN and GaN paves the way for practical assessment of the high-energy electron dynamics for the ternary alloy, AlGaN. (C) 2002 Elsevier Science B.V. All rights reserved.
\end{abstract}

Keywords: Polar optical phonon scattering; Wide band-gap semiconductors; Negative differential conductivity; Brillouin zone integration

\section{Introduction}

AlN with a band gap around $6 \mathrm{eV}$ is practically the widest-gap material that is still considered to be a semiconductor. Wurtzite (WZ) phase of AlN has the further distinction of being the only Al-containing III-V semiconductor with a direct band gap [1]. Even though AlN as a binary compound is rarely used in practical devices, it is an essential compositional part of the $\mathrm{AlGaN} /$

\footnotetext{
*Corresponding author. Tel.: + 44-1206-872-873; fax: + 441206-872-900.

E-mail addresses: bulutay@fen.bilkent.edu.tr (C. Bulutay), bkr@essex.ac.uk (B.K. Ridley).
}

$\mathrm{GaN}$ heterostructures which are currently utilized in high electron mobility transistors (HEMT) working at record high electric fields [2]. AlGaN/ $\mathrm{GaN}$ system offers new prospects both from the technological viewpoint of high-power electronics, and also as a test bed for basic research on highfield transport, where physical understanding of hot electron dynamics at high energies, order of a few electron-volts, is absolutely crucial for the adequate description and modelling of the HEMT's operation. Another reason for our interest in AlN comes from the fact that, among the flourishing III-nitrides, it happens to be the least studied as a bulk system. 
The wide band gap of the III-nitrides that grants the high-field operation is an outcome of the strong ionicity of the chemical bonds [3]. However, the very same ionicity also reflects itself on the enhanced coupling of electrons to polar optical phonons. For AlN, the polar optical phonon (POP) energy is around $112 \mathrm{meV}$ which forms an efficient channel for energy relaxation through successive POP emissions. In this work we analyze the POP scattering in bulk AlN of the conduction band $(\mathrm{CB})$ electrons, tracing them along certain high-symmetry directions. Our present findings complement a similar previous study undertaken by us on $\mathrm{GaN}[4,5]$, and help us to compare these two materials; furthermore, it can aid the characterization of their technologically important alloy, AlGaN. We confine ourselves to the stable WZ phase of AlN, since the metastable zinc-blende phase is very difficult to grow [6] and also has indirect band gap, as was previously predicted [7].

\section{Empirical pseudopotential bandstructure}

For an accurate account of the high-energy electron scattering, we are in need of the band energies and wavefunctions. The empirical pseudopotential method (EPM) is particularly suitable for this purpose, at a reasonable computational cost. The initial task is to gather known data, mainly in the form of band energy separations like the band gap, that will be useful in pinning the band profile. However, compared to GaN, much less experimental data are available for $\mathrm{AlN}$, and also there exists a wide spread over the theoretical values [1]. We base our fitting procedure on the first-principles GW data of Rubio et al., on the band energies [7] and on the conduction band effective mass data of Suzuki et al. [8]. Symmetric $\left(V_{\mathrm{s}}\right)$ and antisymmetric $\left(V_{\mathrm{a}}\right)$ pseudopotential form factors, described by

$$
\begin{aligned}
& V_{\mathrm{s}}(q)=\left(s_{1} q^{3}+s_{2} q^{2}+s_{3}\right) \exp \left(-s_{4} q^{s_{5}}\right), \\
& V_{\mathrm{a}}(q)=\left(a_{1} q^{2}+a_{2} q+a_{3}\right) \exp \left(-a_{4} q^{a_{5}}\right)
\end{aligned}
$$

are optimized in a 10-dimensional parameter space $s_{i}, a_{i}(i=1 \ldots 5)$. This, by itself is a nontrivial task and we made use of a parallel genetic algorithm implemented on a 12-processor SUN HPC 4500 platform [9].

Table 1 lists the fitted parameters and the associated EPM constants that we employed; Fig. 1 shows the corresponding band structure for WZ AlN. We observed an even better performance for this procedure in AlN compared to $\mathrm{GaN}$ which required a post-adjustment of the band gap [4]. The reason for this, possibly, is due to $3 \mathrm{~d}$ orbitals of $\mathrm{Ga}$ atoms whose overlap with $\mathrm{N} 2 \mathrm{~s}$ states requires a nonlocal variant of the EPM [10].

\section{POP scattering rate: theory and results}

The scattering rate due to POP emission based on Fermi's golden rule, considering only normalprocesses is given by

$$
\begin{aligned}
W_{j, m}(\boldsymbol{k})= & \sum_{m^{\prime}} \int \mathrm{d}^{3} k^{\prime} \hat{W}_{j, m^{\prime}}\left(\boldsymbol{k}^{\prime}\right) \delta\left(E_{m^{\prime}}\left(\boldsymbol{k}^{\prime}\right)\right. \\
& \left.-E_{m}(\boldsymbol{k})+\hbar \omega_{j}(\boldsymbol{q})\right), \\
= & \sum_{m^{\prime}} \int_{S} \mathrm{~d} S \frac{\hat{W}_{j, m^{\prime}}\left(\boldsymbol{k}^{\prime}\right)}{\left|\nabla E_{m^{\prime}}\left(\boldsymbol{k}^{\prime}\right)\right|}
\end{aligned}
$$

where

$$
\hat{W}_{j, m^{\prime}}\left(\boldsymbol{k}^{\prime}\right)=\frac{2 \pi}{\hbar} \frac{V}{(2 \pi)^{3}} \Delta_{m^{\prime}, m}\left(\boldsymbol{k}^{\prime}, \boldsymbol{k}\right)\left|C_{j}(\boldsymbol{q})\right|^{2}\left[n_{j}(\vec{q})+1\right] .
$$

Table 1

Fitted values for the parameters in the pseudopotential form factors $V_{\mathrm{s}}$ and $V_{\mathrm{a}}$. These form factors come out in units of $\mathrm{eV}$ when $q$ is used in units of $2 \pi / a$. Other parameters used in EPM are also listed below, where $a$ and $c$ are the lattice constants in the hexagonal plane and along the $c$-axis, respectively, $u$ is the WZ internal structural parameter, and $E_{\max }$ denotes the radius of the energy sphere enclosing the reciprocal lattice vectors

\begin{tabular}{ll}
\hline$S_{1}=0.17349$ & $a_{1}=-0.039177$ \\
$S_{2}=0.082899$ & $a_{2}=0.39083$ \\
$S_{3}=-1.0425$ & $a_{3}=0.60885$ \\
$S_{4}=1.1440$ & $a_{4}=0.79651$ \\
$S_{5}=0.96303$ & $a_{5}=1.8257$ \\
$a=3.110 \AA$ & \\
$c=4.980 \AA$ & \\
$u=0.382$ & \\
$E_{\max }=16 \mathrm{Ry}$ & \\
\hline
\end{tabular}




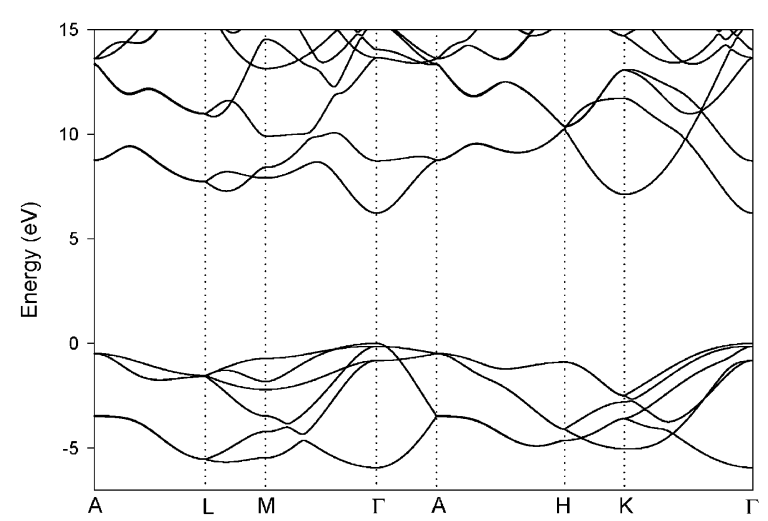

Fig. 1. Band structure obtained using EPM for the WZ phase of AlN; see Table 1 for the associated parameters.

Labels $m, \boldsymbol{k}$ represent the initial-state electron band index and wave vector, respectively; the primed indices correspond to the final-state, after the scattering event. The cell-periodic overlap parameter is given by $\Delta_{m^{\prime}, m}\left(\boldsymbol{k}^{\prime}, \boldsymbol{k}\right)=$ $\left|(1 / \Omega) \| \int_{\Omega} u_{m^{\prime}, k^{\prime}}^{*}(\vec{r}) u_{m, k}(\vec{r}) \mathrm{d}^{3} r\right|^{2}$, where $u_{m, k}(\vec{r})$ is the cell-periodic part of the Bloch function, and $\Omega$ is the volume of the primitive cell. The integration is over the surface $S$, described by the energy conservation relation of one-phonon-emission process, $E_{m^{\prime}}\left(\boldsymbol{k}^{\prime}\right)=E_{m}(\boldsymbol{k})-\hbar \omega_{j}(\boldsymbol{q})$. Here $\hbar \omega_{j}(\boldsymbol{q})$ is the phonon energy corresponding to wave vector $\boldsymbol{q}=\boldsymbol{k}^{\prime}-\boldsymbol{k}$ mapped to first BZ, and $j$ is the polar optical phonon branch index. For the WZ structure the polar phonon branches are longitudinal-optical (LO)-like and transverse-optical (TO)-like [11], whereas in the ZB case it is a pure LO mode. The associated electron-POP coupling coefficients are given by [11]

$$
\begin{aligned}
& \left|C_{\mathrm{LO}-\text { like }}(\boldsymbol{q})\right|^{2} \cong \frac{2 \pi e^{2} \hbar}{V q^{2} \Omega_{\mathrm{L}}\left(\theta_{q}\right)} \\
& \quad \times\left[\frac{\sin ^{2}\left(\theta_{q}\right)}{\left(\frac{1}{\varepsilon_{\perp}^{\infty}}-\frac{1}{\varepsilon_{\perp}^{0}}\right) \omega_{\perp L}^{2}}+\frac{\cos ^{2}\left(\theta_{q}\right)}{\left(\frac{1}{\varepsilon_{z}^{\infty}}-\frac{1}{\varepsilon_{z}^{0}}\right) \omega_{z \mathrm{~L}}^{2}}\right]^{-1}, \\
& \left|C_{\mathrm{TO}-\text { like }}(\boldsymbol{q})\right|^{2}=\frac{2 \pi e^{2} \hbar}{V q^{2} \Omega_{\mathrm{T}}\left(\theta_{q}\right)} \\
& \quad \times \frac{\left(\omega_{\perp}^{2}-\omega_{z}^{2}\right)^{2} \sin ^{2}\left(\theta_{q}\right) \cos ^{2}\left(\theta_{q}\right)}{\left(\varepsilon_{\perp}^{0}-\varepsilon_{\perp}^{\infty}\right) \omega_{\perp}^{2} \cos ^{2}\left(\theta_{q}\right)+\left(\varepsilon_{z}^{0}-\varepsilon_{z}^{\infty}\right) \omega_{z}^{2} \sin ^{2}\left(\theta_{q}\right)},
\end{aligned}
$$

where $V$ is the total crystal volume which cancels in the final expression, $\theta_{q}$ is the angle phonon wave vector $\boldsymbol{q}$ makes with the crystal $c$-axis, $\omega_{\perp}\left(\omega_{z}\right)$ is the TO phonon frequency perpendicular to (along) the $c$-axis, $\omega_{\perp \mathrm{L}}\left(\omega_{z \mathrm{~L}}\right)$ is the LO phonon frequency perpendicular to (along) the $c$-axis, $\varepsilon_{\perp}^{\infty}\left(\varepsilon_{z}^{\infty}\right)$ is the high frequency dielectric constant perpendicular to (along) the $c$-axis. The use of Lyddane-SachsTeller relation gives the static dielectric constants as: $\varepsilon_{\perp}^{0}=\varepsilon_{\perp}^{\infty} \omega_{\perp \mathrm{L}}^{2} / \omega_{\perp}^{2}$ and $\varepsilon_{z}^{0}=\varepsilon_{z}^{\infty} \omega_{z \mathrm{~L}}^{2} / \omega_{z}^{2}$. The angle-dependent phonon frequencies are given by [11]

$$
\begin{aligned}
& \Omega_{\mathrm{L}}\left(\theta_{q}\right)=\sqrt{\omega_{z \mathrm{~L}}^{2} \cos ^{2} \theta_{q}+\omega_{\perp \mathrm{L}}^{2} \sin ^{2} \theta_{q}}, \\
& \Omega_{\mathrm{T}}\left(\theta_{q}\right)=\sqrt{\omega_{z}^{2} \sin ^{2} \theta_{q}+\omega_{\perp}^{2} \cos ^{2} \theta_{q}} .
\end{aligned}
$$

We use the following data [12] for the WZ AlN phonons: $\hbar \omega_{z}=76.1 \mathrm{meV}, \hbar \omega_{z \mathrm{~L}}=110.7 \mathrm{meV}$, $\hbar \omega_{\perp}=83.4 \mathrm{meV}, \quad \hbar \omega_{\perp \mathrm{L}}=113.6 \mathrm{meV}$, and $\varepsilon_{\perp}^{\infty}=$ $\varepsilon_{z}^{\infty}=4.68$. Note that optical phonon energy is about 4.5 times the thermal energy $(\sim 25 \mathrm{meV})$ at room temperature. For this reason, phonon occupation probability, $n_{j}(\boldsymbol{q})$, will be negligible up to room temperature, so that we can essentially set $n_{j}(\boldsymbol{q}) \equiv 0$. With the same reasoning we ignore the POP absorption processes in the electron scattering.

The Lehmann-Taut BZ integration technique [13] is very suitable for the computation of the scattering rate expression shown in Eq. (1). Accordingly, we divide the irreducible wedge of the first BZ into fine tetrahedra, and store the band energies and the cell-periodic overlap parameters at the nodes of these tetrahedra. Other details of our technique can be found in Ref. [4].

Starting from the $\mathrm{CB}$ minimum at the $\Gamma$ point, we trace the POP scattering rates of the $\mathrm{CB}$ electrons, assuming a very high electric field applied along a high-symmetry line: $\Gamma-\mathrm{M}, \Gamma-\mathrm{K}$, $\Gamma-\mathrm{A}$ and $\Gamma-\mathrm{U}_{\text {min }}$. Due to BZ folding along $\Gamma-\mathrm{A}$ direction [4], we actually follow an extended trajectory as $\Gamma_{1}-\mathrm{A}-\Gamma_{3}$. The results are shown in Fig. 2; only the LO-like POP branch is considered, as the TO-like scattering rate was found to be two orders of magnitude smaller [4]. Satellite valleys play a major role in the quantitative value of the scattering rates: as soon as scattering to other 

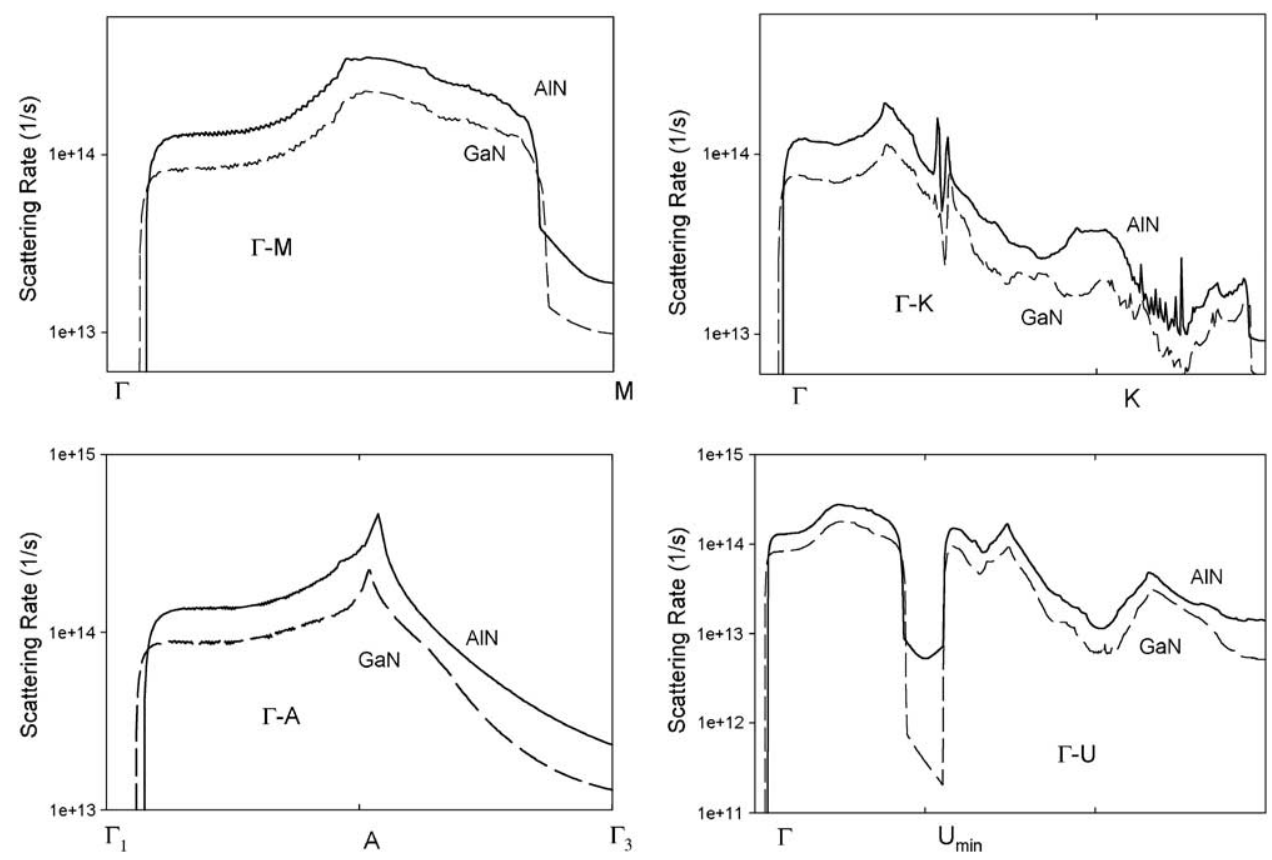

Fig. 2. POP scattering rates of $\mathrm{CB}$ electrons for $\mathrm{WZ} \mathrm{AlN}$ (solid) and $\mathrm{GaN}$ (dashed) along the directions $\Gamma-\mathrm{M}, \Gamma-\mathrm{K}, \Gamma-\mathrm{A}$ and $\Gamma-\mathrm{U}_{\mathrm{min}}$. Due to BZ folding along $\Gamma-\mathrm{A}$ direction an extended trajectory, $\Gamma_{1}-\mathrm{A}-\Gamma_{3}$, is followed.

satellite valleys becomes energetically possible, the scattering rate significantly increases from its band edge value. For comparison, results for the WZ phase of GaN are also included. AlN is seen to possess about $50 \%$ higher POP scattering rate than GaN. This is in line with the higher ionicity of AlN if the more recent García and Cohen [3] scale is used, whereas according to Phillips ionicity scale [14] the opposite was predicted.

\section{Esaki-Tsu estimation for high-field drift velocity}

In the case of GaN, we have compared the POP rate with an overall non-polar deformation potential scattering, and observed the former to be by far dominant for the energies within about $2.5 \mathrm{eV}$ of the $\mathrm{CB}$ minimum, which is typically the bandwidth of the lowest $\mathrm{CB}$ in all directions [4]. Anticipating AlN to obey a similar trend for the energy range of concern, we can discard other scattering mechanisms. Based on these POP scattering rates, we estimate the high-field drift velocity behaviour using Esaki and Tsu's phenomenological semiclassical treatment [15], where the average drift velocity is given by

$v_{\mathrm{d}}=e F \hbar^{-2} \int_{0}^{\infty} E^{\prime \prime} \mathrm{e}^{-t / \tau} \mathrm{d} t$,

here $E^{\prime \prime}$ is the curvature of the energy band diagram along the applied field's direction $\boldsymbol{F}$, sampled at the point $\boldsymbol{k}(t)=e \boldsymbol{F} t / \hbar$, for an electron originating from the $\Gamma$ point. For the scattering time $\tau$ in this equation, we use the value given by the maximum scattering rate in each direction, monitoring the full period of the electron's trajectory in the wave vector-space (for certain directions, exceeding the first BZ boundary). We would like to stress that such an approach makes sense only at very high electric fields, say, above $0.5 \mathrm{MV} / \mathrm{cm}$. With this proviso, we provide in Fig. 3 the corresponding drift velocity behavior along several high-symmetry directions for WZ AIN and $\mathrm{GaN}$. Electrons reaching the negative effective 


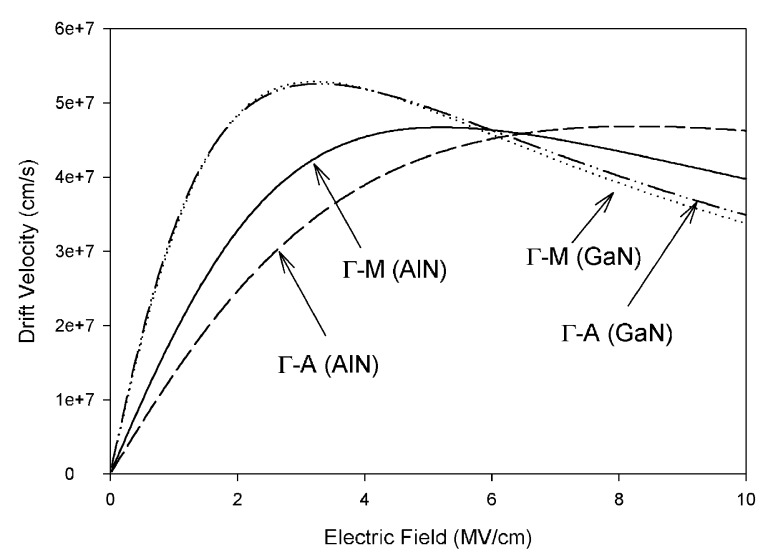

Fig. 3. High-field-related drift velocity versus electric field estimations along several directions for WZ AIN and GaN.

mass part of the band structure before undergoing a scattering event, give rise to a negative differential conductivity (NDC). As this idea was initially conjectured by Krömer [16] and later applied to superlattices by Esaki and Tsu [15], it can be distinguished from other NDC mechanisms by Krömer-Esaki-Tsu NDC. The effect is seen to require quite high electric fields, exceeding a few $\mathrm{MV} / \mathrm{cm}$. With a substantially higher POP scattering rate, AlN has a much larger onset field for the NDC compared to GaN. Whether such an effect can be observed before the breakdown of the material is further questionable. Both experiments and a more refined theoretical analysis will be appropriate to check this phenomenological estimation.

\section{Conclusions}

We have presented a full-band POP scattering analysis of $\mathrm{CB}$ electrons for the WZ crystal phase of AlN by carrying out a directional assessment throughout the extent of the Brillouin zone. Higher ionicity of AlN compared to GaN results in typically a $50 \%$ increase in the POP scattering rate. Our results will be particularly important for researchers working on full-band Monte Carlo simulation of hot electron effects in wide band gap semiconductors and high-power HEMT devices. Based on our preliminary estimations, onset fields for observing the anticipated Krömer-Esaki-Tsu NDC is substantially higher for AIN than GaN. Availability of these results leads to the practical assessment of the high energy electron dynamics for the important ternary alloy, AlGaN.

\section{Acknowledgements}

Work of CB is supported by The Scientific and Technical Research Council of Turkey (TÜBITAK); work of BKR is supported by ONR (N00014-99-1-0014).

\section{References}

[1] I. Vurgaftman, J.R. Meyer, L.R. Ram-Mohan, J. Appl. Phys. 89 (2001) 5815.

[2] L.F. Eastman, Phys. Stat. Sol. A 176 (1999) 175.

[3] A. García, M.L. Cohen, Phys. Rev. B 47 (1993) 4215; A. García, M.L. Cohen, Phys. Rev. B 47 (1993) 4221.

[4] C. Bulutay, B.K. Ridley, N.A. Zakhleniuk, Phys. Rev. B 62 (2000) 15754.

[5] C. Bulutay, B.K. Ridley, N.A. Zakhleniuk, Appl. Phys. Lett. 77 (2000) 2707.

[6] M.P. Thompson, G.W. Auner, T.S. Zheleva, K.A. Jones, S.J. Simko, J.N. Hilfiker, J. Appl. Phys. 89 (2001) 3331.

[7] A. Rubio, J.L. Corkill, M.L Cohen, E.L. Shirley, S.G. Louie, Phys. Rev. B 48 (1993) 11810.

[8] M. Suzuki, T. Uenoyama, A. Yanase, Phys. Rev. B 52 (1995) 8132.

[9] C. Kirman, C. Bulutay, unpublished.

[10] M. Goano, E. Bellotti, E. Ghillino, G. Ghione, K. Brennan, J. Appl. Phys. 88 (2000) 6467.

[11] B.C. Lee, K.W. Kim, M. Dutta, M.A. Stroscio, Phys. Rev. B 56 (1997) 997.

[12] J.C. Nipko, C.-K. Loong, Phys. Rev. B 57 (1998) 10550.

[13] G. Lehmann, M. Taut, Phys. Stat. Sol. B 54 (1972) 469.

[14] J.C. Phillips, Rev. Mod. Phys. 42 (1970) 317.

[15] L. Esaki, R. Tsu, IBM J. Res. Dev. 14 (1970) 61.

[16] H. Krömer, Phys. Rev. 109 (1958) 1856. 